\title{
Control Strategy for Transition Flight of a Fixed-wing UAV*
}

\author{
By Ryota HATORI, Satoshi KonO and Kenji UCHIYAMA \\ Department of Aerospace Engineering, Nihon University, Funabashi, Japan
}

(Received January $\left.16^{\text {th }}, 2014\right)$

\begin{abstract}
We propose a control system for a small fixed-wing unmanned aerial vehicle (UAV) transitioning from level flight to hovering without switching the controller gains and dynamical model. The proposed system is divided into translational and rotational control systems. Nonlinear equations of motion are linearized using a dynamic inversion method in the flight control system design. A robust rotational motion controller is employed because the attitude of the UAV is significantly affected by disturbances such as wind. During the rotational controller design, the quaternion system was employed in place of an Euler angle to express the attitude angle of the UAV. This method enables the successive elimination of nonlinear terms through nonlinear state feedback because it is not necessary to select a typical equilibrium point. The unknown parameters of the nonlinear equations and the velocity of the UAV are estimated by an observer that is based on the disturbanceaccommodating control (DAC) method, and an extended Kalman filter, respectively. We have confirmed the effectiveness of the proposed flight control system through numerical simulations and experiments.
\end{abstract}

Key Words: Fixed-wing UAV, Transition Flight, Level Flight, Hovering, Dynamic Inversion Method

\section{Introduction}

Unmanned aerial vehicles (UAV) are being developed all over the world ${ }^{1-4)}$ and are now used for missions such as disaster monitoring, crop spraying, and atmospheric observation. Hitherto UAVs have roughly been classified as either a fixedwing type or rotary-wing type. Fixed-wing UAVs are able to travel at a high speed with low fuel consumption in comparison to rotary-wing UAVs, making them suitable for missions that demand rapid coverage of very large areas. On the other hand, rotary-wing UAVs can hold a fixed position, meaning they can observe a stationary target to which access is difficult, such as a disaster zone.

Therefore, an UAV that combines the advantages of both types is required for fixed-point observation missions in which long distances must be traveled. Some researchers have investigated fixed-wing UAVs that can transition from level flight to hovering. The UAV of a complicated structure is classified as tilt wing, ${ }^{5-6)}$ tilt rotor UAV, ${ }^{7}$ or tail sitter UAVs. ${ }^{8-10)}$ Their complicated structure influences reliability and cost with respect to maintenance and manufacturing. It would be difficult to keep a controlled performance due to nonlinearity that is caused by the structure. For a propeller-driven tail-sitter, slipstream flow over aircraft control surfaces is a crucial problem for stabilization during the transition flight. This work addresses the transition from level flight to hovering performed by fixedwing UAVs. Myrand-Lapierre et al. achieved the transition by switching the control gains and the linear motion model. ${ }^{11)}$ However, the stability of the flight system during the transition is not guaranteed because of the nonlinearity of the dynamics.

(C) 2014 The Japan Society for Aeronautical and Space Sciences

*Presented at the 2013 Asia-Pacific International Symposium on Aerospace

Technology (APISAT-2013), Nov. 20-22, 2013, Takamatsu, Japan
Achieving stability during the transition period poses a complex issue.

We propose a flight control system that does not require switching of the controller gains and dynamical model. In place of an Euler angle, the quaternion system is employed to express the attitude angle of a fixed-wing UAV. Nonlinear equations of motion are linearized using a dynamic inversion method ${ }^{12)}$ to design a flight control system. A robust controller is applied to the linearized system, making the UAV robust to the effects of wind. The unknown parameters of the nonlinear equations and the velocity of the UAV are estimated by an observer based on the disturbanceaccommodating control (DAC) method ${ }^{13)}$ and an extended Kalman filter ${ }^{14)}$ respectively. We confirm the effectiveness of the proposed flight control system through numerical simulations and experiments.

\section{Dynamics}

\subsection{Attitude description}

An inertial coordinate system and a body coordinate system are used. $U, V$ and $W$ are the velocities along the $x_{B}, y_{B}$, and $z_{B}$ axes, respectively. $p, q$ and $r$ are the angular velocities around each axis. The deflection angles of the control surfaces are denoted by $\delta_{a}, \delta_{e}$ and $\delta_{r}$ for the ailerons, elevators and rudder, respectively. The nose mounted-propeller generates thrust $\mathbf{T}$. 
Body coordinate system
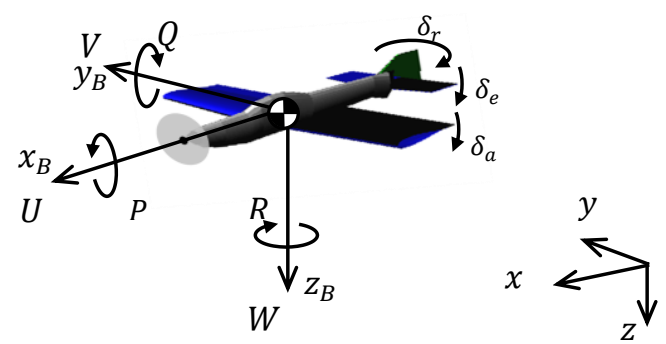

Inertial coordinate system

Fig. 1. Coordinate systems and parameter definitions

\subsection{Equations of motion}

The nonlinear equations of motion are defined as follows:

$$
\begin{gathered}
\dot{\mathbf{V}}=-\widetilde{\boldsymbol{\omega}} \mathbf{V}+\mathbf{C}^{B / I} \mathbf{g}+\frac{1}{m}\{\mathbf{C}(\alpha) \mathbf{F}+\mathbf{T}\} \\
\dot{\boldsymbol{\omega}}=-\mathbf{J}^{-\mathbf{1}} \widetilde{\boldsymbol{\omega}} \mathbf{J} \boldsymbol{\omega}+\mathbf{J}^{-\mathbf{1}} \mathbf{M}+\mathbf{J}^{-\mathbf{1}} \mathbf{M}_{u} \mathbf{u}
\end{gathered}
$$

where

$$
\begin{aligned}
& \mathbf{V}=\left[\begin{array}{lll}
U & V & W
\end{array}\right]^{T}, \boldsymbol{\omega}=\left[\begin{array}{lll}
P & Q & R
\end{array}\right]^{T}, \mathbf{g}=\left[\begin{array}{lll}
0 & 0 & g
\end{array}\right]^{T} \\
& \mathbf{T}=\left[\begin{array}{lll}
T & 0 & 0
\end{array}\right]^{T}, \mathbf{u}=\left[\begin{array}{lll}
\delta_{a} & \delta_{e} & \delta_{r}
\end{array}\right]^{T} \\
& \widetilde{\boldsymbol{\omega}}=\left[\begin{array}{ccc}
0 & -R & Q \\
R & 0 & -P \\
-Q & P & 0
\end{array}\right], \mathbf{F}=\bar{q} S\left[\begin{array}{c}
-C_{D}(\alpha) \\
C_{y}(\beta)+C_{y R} R+C_{y \delta_{r}} \delta_{r} \\
C_{L}(\alpha)+C_{L \delta_{e}} \delta_{e}
\end{array}\right] \\
& \mathbf{C}(\alpha)=\left[\begin{array}{ccc}
\cos (\alpha) & 0 & -\sin (\alpha) \\
0 & 1 & 0 \\
\sin (\alpha) & 0 & \cos (\alpha)
\end{array}\right], \mathbf{J}=\left[\begin{array}{ccc}
I_{x x} & 0 & -I_{x z} \\
0 & I_{y y} & 0 \\
-I_{x z} & 0 & I_{z z}
\end{array}\right] \\
& \mathbf{M}=\bar{q} S\left[\begin{array}{c}
b\left\{C_{l}(\beta)+C_{l P} P+C_{l R} R\right\} \\
c\left\{C_{m}(\alpha)+C_{m}(\dot{\alpha})+C_{m_{Q}} Q\right\} \\
b\left\{C_{n}(\beta)+C_{n P} P+C_{n R} R\right\}
\end{array}\right]+\left[\begin{array}{c}
-M_{\text {drag }}-I_{\text {prop }} \dot{\omega}_{\text {prop }} \\
-I_{\text {prop }} \omega_{\text {prop }} R \\
I_{\text {prop }} \omega_{\text {prop }} Q
\end{array}\right] \\
& \mathbf{M}_{u}=\bar{q} S\left[\begin{array}{ccc}
b C_{l \delta_{a}} & 0 & b C_{l \delta_{r}} \\
0 & \bar{c} C_{m \delta_{e}} & 0 \\
b C_{n \delta_{a}} & 0 & b C_{n \delta_{r}}
\end{array}\right]+\bar{q}_{\text {prop }} S\left[\begin{array}{ccc}
d C_{l \delta_{a}} & 0 & d C_{l \delta_{r}} \\
0 & \bar{c} C_{m \delta_{e}} & 0 \\
d C_{n \delta_{a}} & 0 & d C_{n \delta_{r}}
\end{array}\right] \\
& \mathbf{C}^{B / I}=\left[\begin{array}{ccc}
q_{1}{ }^{2}-q_{2}{ }^{2}-q_{3}{ }^{2}+q_{4}{ }^{2} & 2\left(q_{1} q_{2}+q_{3} q_{4}\right) & 2\left(q_{3} q_{2}-q_{2} q_{4}\right) \\
2\left(q_{1} q_{2}-q_{3} q_{4}\right) & q_{2}^{2}-q_{3}{ }^{2}-q_{1}{ }^{2}+q_{4}{ }^{2} & 2\left(q_{2} q_{3}-q_{1} q_{4}\right) \\
2\left(q_{3} q_{1}-q_{2} q_{4}\right) & 2\left(q_{2} q_{3}-q_{1} q_{4}\right) & q_{3}{ }^{2}-q_{1}^{2}-q_{2}{ }^{2}+q_{4}{ }^{2}
\end{array}\right]
\end{aligned}
$$

Here $\alpha$ and $\beta$ are the angle of attack and sideslip, respectively. $S, \overline{\mathbf{C}}$ and $\mathbf{J}$ denote the wing area, mean aerodynamic chord and inertia tensor, respectively. The subscript "prop" indicates physical quantities regarding the propeller. $\bar{q}$ is the dynamic pressure applied to the aircraft during flight, and $\bar{q}_{\text {prop }}$ is the dynamic pressure generated in the propeller slipstream. $\mathbf{C}^{B / I}$ is the transformation matrix using the quaternion system. The aerodynamic coefficients in terms of the angle of attack are denoted by $C_{L}(\alpha), C_{D}(\alpha)$ and $C_{m}(\alpha)$.

\section{Flight Control System ${ }^{15-16)}$}

The proposed flight control system consists of translational and rotational motion controllers. The dynamic inversion method is used to linearize the nonlinear dynamics of the UAV in the design of both controllers. Figure 2 shows a block diagram of the proposed flight control system.

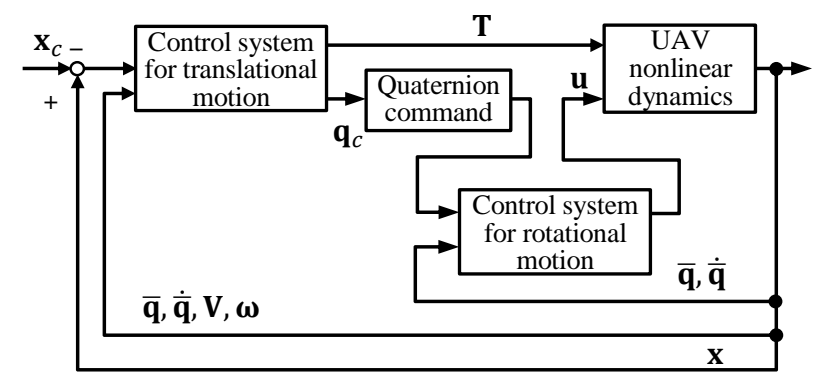

Fig. 2. Block diagram of the flight control system

\subsection{Translational controller}

We define the position error $\mathbf{x}_{e}$ as shown in the following equation. $\mathbf{x}$ is the current position of the UAV and $\mathbf{x}_{c}$ the desired position.

$$
\mathbf{x}_{e}=\mathbf{x}-\mathbf{x}_{c}=\left[\begin{array}{lll}
x-x_{c} & y-y_{c} & z-z_{c}
\end{array}\right]^{T}
$$

The acceleration of the UAV can be expressed using the transformation matrix $\mathbf{C}^{I / B}$ and the velocity vector $\mathbf{V}$ as follows:

$$
\ddot{\mathbf{x}}_{e}=\dot{\mathbf{C}}^{I / B} \mathbf{V}+\mathbf{C}^{I / B} \dot{\mathbf{V}}
$$

The equation of translational motion for the UAV is derived from Eqs. (1) and (4).

$$
\begin{aligned}
\ddot{\mathbf{x}}_{e} & =\dot{\mathbf{C}}^{I / B} \mathbf{V}-\mathbf{C}^{I / B} \widetilde{\boldsymbol{\omega}} \mathbf{V}-\mathbf{g}-\frac{1}{m} \mathbf{C}^{I / B} \mathbf{C}(\alpha) \mathbf{F}+\frac{1}{m} \mathbf{T}^{I / B} \\
& =\mathbf{z}_{t 1}+\frac{1}{m} \mathbf{T}^{I / B}
\end{aligned}
$$

Here $\mathbf{T}^{I / B}=\mathbf{C}^{I / B} \mathbf{T}=\left[\begin{array}{lll}T_{x} & T_{y} & T_{z}\end{array}\right]^{\boldsymbol{T}}$ is the thrust vector in the inertial coordinate system. The first term on the right-hand side in Eq. (5) $\boldsymbol{z}_{t 1}$ is an unknown parameter that includes aerodynamic force, $\mathbf{F}$. It is not necessary to apply a parametric estimation method to obtain the value of this parameter. Therefore, we apply a DAC observer, which is a nonparametric estimation method, to the translational motion controller. The unknown parameter is defined as a polynomial of time $t$.

$$
\mathbf{z}_{t 1}=\mathbf{c}_{1} t+\mathbf{c}_{0}, \quad \mathbf{z}_{t 2}=\dot{\mathbf{z}}_{t 1}=\mathbf{c}_{1}, \quad \mathbf{z}_{t 3}=\dot{\mathbf{z}}_{t 2}=0
$$

Equation (6) can be rewritten in terms of the estimates using the DAC observer.

$$
\ddot{\hat{\mathbf{x}}}_{e}=\hat{\mathbf{z}}_{t 1}+\frac{1}{m} \mathbf{T}^{I / B}+\mathbf{L}_{t}\left(\dot{\mathbf{x}}_{e}-\dot{\hat{\mathbf{x}}}_{e}\right)
$$

Here $\mathbf{L}_{t}$ is an observer gain. The hat symbol denotes an estimator of the parameter. Using Eqs. (6) and (7), a DAC observer is constructed as represented by the following equation. 


$$
\begin{aligned}
& \frac{d}{d t}\left[\begin{array}{c}
\hat{\mathbf{\mathbf { x }}}_{e} \\
\hat{\mathbf{z}}_{t 1} \\
\hat{\mathbf{z}}_{t 2}
\end{array}\right]=\left[\begin{array}{lll}
\mathbf{0}_{3 \times 3} & \mathbf{I}_{3 \times 3} & \mathbf{0}_{3 \times 3} \\
\mathbf{0}_{3 \times 3} & \mathbf{0}_{3 \times 3} & \mathbf{I}_{3 \times 3} \\
\mathbf{0}_{3 \times 3} & \mathbf{0}_{3 \times 3} & \mathbf{0}_{3 \times 3}
\end{array}\right]+\frac{1}{m}\left[\begin{array}{c}
\mathbf{I}_{3 \times 3} \\
\mathbf{0}_{3 \times 3} \\
\mathbf{0}_{3 \times 3}
\end{array}\right] \mathbf{T}^{I / B} \\
& +\left[\begin{array}{l}
\mathbf{L}_{t 1} \\
\mathbf{L}_{t 2} \\
\mathbf{L}_{t 3}
\end{array}\right]\left(\dot{\mathbf{x}}_{e}-\dot{\hat{\mathbf{x}}}_{e}\right)
\end{aligned}
$$

When the DAC observer works well, the thrust $\mathbf{T}^{I / B}$ is expressed by the following equation.

$$
\mathbf{T}^{I / B}=m\left(-\hat{\mathbf{z}}_{t 1}+\mathbf{v}_{x}\right)
$$

where $\boldsymbol{v}_{x}$ is regarded as a new input of the linearized system. The equation of translational motion is rewritten as a matrix of differential equations.

$$
\frac{d}{d t}\left[\begin{array}{l}
\mathbf{x}_{e} \\
\dot{\mathbf{x}}_{e}
\end{array}\right]=\left[\begin{array}{ll}
\mathbf{0}_{3 \times 3} & \mathbf{I}_{3 \times 3} \\
\mathbf{0}_{3 \times 3} & \mathbf{0}_{3 \times 3}
\end{array}\right]\left[\begin{array}{l}
\mathbf{x}_{e} \\
\dot{\mathbf{x}}_{e}
\end{array}\right]+\left[\begin{array}{l}
\mathbf{0}_{3 \times 3} \\
\mathbf{I}_{3 \times 3}
\end{array}\right] \mathbf{v}_{x}
$$

Here, $\mathbf{v}_{x}$ is defined as the proposal derivative controller in this study.

$$
\mathbf{v}_{x}=\mathbf{k}_{t 1} \mathbf{x}_{e}+\mathbf{k}_{t 2} \dot{\mathbf{x}}_{e}
$$

$\mathbf{k}_{t}$ is the feedback gain. The amplitude of thrust $T$, is calculated using the following equation.

$$
T=\sqrt{T_{x}^{2}+T_{y}^{2}+T_{z}^{2}}
$$

Measurements from sensors such as a GPS or an IMU normally contain an observation error. In addition, it is necessary to obtain the ground speed of a UAV to design a flight control system. In order to overcome these issues, an extended Kalman filter is applied to estimate the current position $\mathbf{x}$ and its time derivative $\dot{\mathbf{x}}$, as shown in Fig. 3 .

$$
\begin{gathered}
\mathbf{x}_{k+1}=\mathbf{F} \mathbf{x}_{k}+\mathbf{w}_{k} \\
\mathbf{y}_{k}=\mathbf{H} \mathbf{x}_{k}+\mathbf{v}_{k}
\end{gathered}
$$

Here $\mathbf{w}_{k}$ is the measurement noise and $\mathbf{v}_{k}$ is the system noise. It is assumed that these noises conform to a normal distribution. The state variable $\mathbf{x}_{k}$ and the matrices $\mathbf{F}$ and $\mathbf{H}$ in Eq. (13) can be expressed as

$$
\begin{gathered}
\mathbf{x}_{k}=[x(k) y(k) z(k) \dot{x}(k) \dot{y}(k) \dot{z}(k) \ddot{x}(k) \ddot{y}(k) \ddot{z}(k)]^{T} \\
\mathbf{F}=\left[\begin{array}{lcc}
\mathbf{I}_{3 \times 3} & \Delta T \times \mathbf{I}_{3 \times 3} & \frac{\Delta T^{2}}{2} \times \mathbf{I}_{3 \times 3} \\
\mathbf{0}_{3 \times 3} & \mathbf{I}_{3 \times 3} & \Delta T \times \mathbf{I}_{3 \times 3} \\
\mathbf{0}_{3 \times 3} & \mathbf{0}_{3 \times 3} & \mathbf{I}_{3 \times 3}
\end{array}\right] \\
\mathbf{H}=\left[\begin{array}{lll}
\mathbf{I}_{3 \times 3} & \mathbf{0}_{3 \times 3} & \mathbf{0}_{3 \times 3} \\
\mathbf{0}_{3 \times 3} & \mathbf{0}_{3 \times 3} & \mathbf{0}_{3 \times 3} \\
\mathbf{0}_{3 \times 3} & \mathbf{0}_{3 \times 3} & \mathbf{I}_{3 \times 3}
\end{array}\right]
\end{gathered}
$$

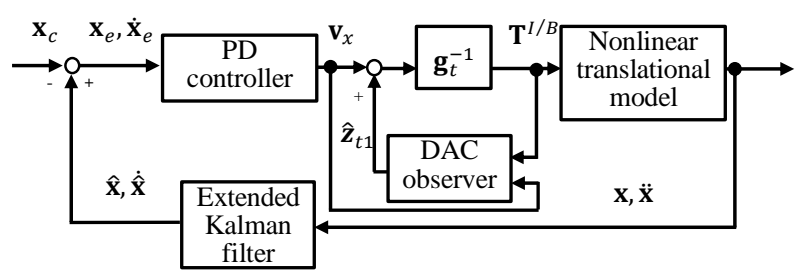

Fig. 3. Block diagram for controlling translational motion

\subsection{Rotational controller}

The attitude control of the UAV is the most important issue for transitional flight. Therefore, a robust controller is designed for the rotational motion because the UAV is significantly affected by disturbances such as wind.

The command signal to the controller is generated such that the thrust direction of the UAV agrees with the thrust vector $\mathbf{T}^{I / B}$. Pitch angle $\theta_{c}$ and heading angle $\psi_{c}$ commands can be defined using components of the thrust vector as given in the following equations.

$$
\theta_{c}=\tan ^{-1}\left(\frac{T_{z}}{T_{x}}\right), \psi_{c}=\sin ^{-1}\left(\frac{T_{z}}{T}\right)
$$

It is necessary to transform each command angle in Eq.(6) into a quaternion command $\mathbf{q}_{c}$ that is calculated using Eqs. (16) to (18).

$$
\begin{aligned}
& \mathbf{q}_{\theta}=\left[\begin{array}{llll}
0 & \sin \left(\frac{\theta_{c}}{2}\right) & 0 & \cos \left(\frac{\theta_{c}}{2}\right)
\end{array}\right]^{T}
\end{aligned}
$$

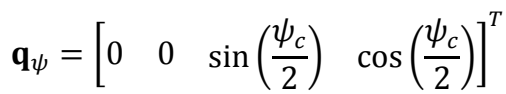

$$
\begin{aligned}
& \mathbf{q}_{c}=\left[\begin{array}{c}
q_{c 1} \\
q_{c 2} \\
q_{c 3} \\
q_{c 4}
\end{array}\right]=\left[\begin{array}{cccc}
q_{\theta 4} & -q_{\theta 3} & q_{\theta 2} & q_{\theta 1} \\
q_{\theta 3} & q_{\theta 4} & -q_{\theta 1} & q_{\theta 2} \\
-q_{\theta 2} & q_{\theta 1} & q_{\theta 4} & q_{\theta 3} \\
-q_{\theta 1} & -q_{\theta 2} & -q_{\theta 3} & q_{\theta 4}
\end{array}\right]\left[\begin{array}{l}
q_{\psi 1} \\
q_{\psi 2} \\
q_{\psi 3} \\
q_{\psi 4}
\end{array}\right]
\end{aligned}
$$

The control system for the rotational motion is similar in configuration to that of the translational controller. The kinematics relating to the quaternion can be expressed using an angular velocity vector $\boldsymbol{\omega}$

$$
\begin{aligned}
& \ddot{\overline{\mathbf{q}}}=\frac{1}{2} \dot{\overline{\mathbf{E}}}(\mathbf{q}) \boldsymbol{\omega}+\frac{1}{2} \overline{\mathbf{E}}(\mathbf{q}) \dot{\boldsymbol{\omega}} \\
& \overline{\mathbf{E}}(\mathbf{q})=\left[\begin{array}{ccc}
q_{4} & -q_{3} & q_{2} \\
q_{3} & q_{4} & -q_{1} \\
-q_{2} & q_{1} & q_{4}
\end{array}\right]
\end{aligned}
$$

The equations of rotational motion for the UAV can be derived from Eq. (20).

$$
\begin{aligned}
\ddot{\mathbf{q}} & =\frac{1}{2}\left\{\dot{\overline{\mathbf{E}}}(\mathbf{q}) \omega-\overline{\mathbf{E}}(\mathbf{q}) \mathbf{J}^{-1} \widetilde{\boldsymbol{\omega}} \mathbf{J} \omega+\overline{\mathrm{E}}(\mathbf{q}) \mathbf{J}^{-1} \mathbf{M}\right\} \\
& +\frac{1}{2} \overline{\mathrm{E}}(\mathbf{q}) \mathbf{J}^{-1} \mathbf{M}_{u} \mathbf{u} \\
& =\mathbf{z}_{r 1}+\mathbf{g}_{r} \mathbf{u}
\end{aligned}
$$

where $\mathbf{g}_{r}$ denotes the coefficient matrix of the control input $\mathbf{u}$. The unknown parameter $\mathbf{z}_{r 1}$ is estimated by the DAC observer in the same way as that in Eq. (5). Similarly, the unknown parameter in Eq. (21) is defined as a polynomial of time $t$. 


$$
\mathbf{z}_{r 1}=\mathbf{c}_{r 1} \mathrm{t}+\mathbf{c}_{r 0}, \mathbf{z}_{r 2}=\dot{\mathbf{z}}_{r 1}=\mathbf{c}_{r 1}, \mathbf{z}_{r 3}=\dot{\mathbf{z}}_{r 2}=0
$$

Equation (21) can be rewritten in terms of the estimates using the DAC observer.

$$
\ddot{\widehat{\mathbf{q}}}=\hat{\mathbf{z}}_{r 1}+\mathbf{g}_{r 1} \mathbf{T}^{I / B}+\mathbf{L}_{r}(\dot{\overline{\mathbf{q}}}-\dot{\hat{\mathbf{q}}})
$$

The control input $\mathbf{u}$ is expressed using an estimated value.

$$
\mathbf{u}=\mathbf{g}_{r}^{-1}\left(-\hat{\mathbf{z}}_{r 1}+\mathbf{v}_{q}\right)
$$

Here $\boldsymbol{v}_{q}$ is a new control input for the linearized dynamics. The equation of rotational motion is rewritten as a matrix of differential equations.

$$
\frac{d}{d t}\left[\begin{array}{c}
\overline{\mathbf{q}} \\
\dot{\mathbf{q}}
\end{array}\right]=\left[\begin{array}{cc}
\mathbf{0}_{3 \times 3} & \mathbf{I}_{3 \times 3} \\
\mathbf{0}_{3 \times 3} & \mathbf{0}_{3 \times 3}
\end{array}\right]\left[\begin{array}{c}
\overline{\mathbf{q}} \\
\dot{\mathbf{q}}
\end{array}\right]+\left[\begin{array}{c}
\mathbf{0}_{3 \times 3} \\
\mathbf{I}_{3 \times 3}
\end{array}\right] \mathbf{v}_{q}
$$

If the estimation error of the DAC observer is not sufficiently small, the nonlinear term is not completely canceled when using the dynamic inversion method. Then the error is treated as an uncertainty, as shown in Eq. (26).

$$
\begin{gathered}
\ddot{\overline{\mathbf{q}}}=\mathbf{z}_{r 1}+\mathbf{g}_{r} \mathbf{g}_{r}^{-1}\left(-\hat{\mathbf{z}}_{r 1}+\mathbf{v}_{q}\right) \\
=\left(\mathbf{z}_{r 1}-\hat{\mathbf{z}}_{r 1}\right)+\mathbf{v}_{q} \\
=\Delta+\mathbf{v}_{q} \\
\frac{d}{d t}\left[\begin{array}{c}
\overline{\mathbf{q}} \\
\dot{\overline{\mathbf{q}}}
\end{array}\right]=\left[\begin{array}{cc}
\mathbf{0}_{3 \times 3} & \mathbf{I}_{3 \times 3} \\
\mathbf{0}_{3 \times 3} & \mathbf{0}_{3 \times 3}
\end{array}\right]\left[\begin{array}{c}
\overline{\mathbf{q}} \\
\dot{\overline{\mathbf{q}}}
\end{array}\right]+\left[\begin{array}{c}
\mathbf{0}_{3 \times 3} \\
\mathbf{I}_{3 \times 3}
\end{array}\right] \mathbf{v}_{q}+\left[\begin{array}{c}
\mathbf{0}_{3 \times 3} \\
\mathbf{I}_{3 \times 3}
\end{array}\right] \Delta
\end{gathered}
$$

The $\mathrm{H}_{\infty}$ controller is designed to suppress not only the influence of the uncertainty in Eqs. (26) and (27), but also disturbances such as wind. This is achieved by solving the linear matrix inequality (LMI) in this study. The control input is expressed by the following equation.

$$
\mathbf{v}_{q}=K(s)\left[\begin{array}{l}
\overline{\mathbf{q}}_{e} \\
\dot{\mathbf{q}}_{e}
\end{array}\right]
$$

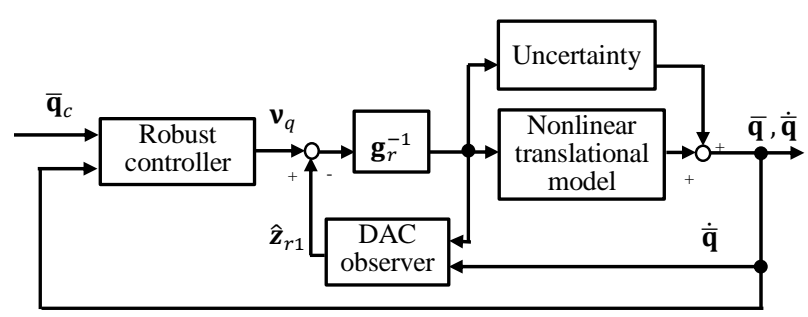

Fig. 4. Block diagram for control of rotational motion

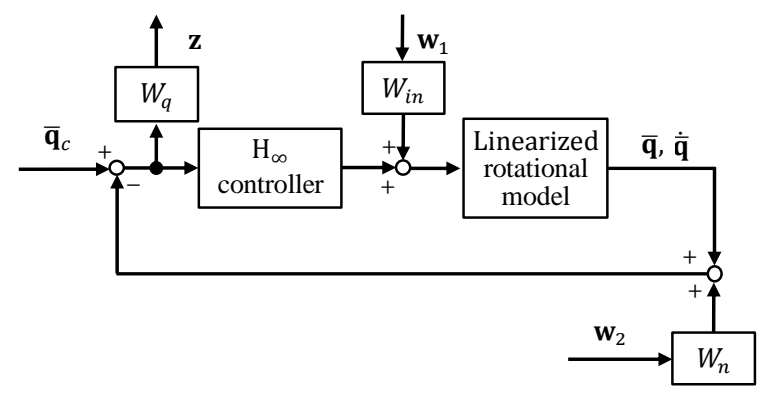

Fig. 5. Block diagram of robust controller
Figure 4 shows a block diagram for the rotational motion control of the UAV. The details of the robust controller are shown in Fig. 5. $W_{i n}$ is weighting functions for multiplicative uncertainty. $W_{n}$ and $W_{q}$ are weighting functions for the sensor noise and the error between the command quaternion $\overline{\mathbf{q}}_{c}$ and the current quaternion $\overline{\mathbf{q}}$, respectively.

\section{Numerical Simulation}

First, we examined the performance of the proposed flight control system through numerical simulations. Aerodynamic coefficients, stability derivatives and control derivatives in the numerical simulation are derived by DATCOM (data compendium). The moments of inertia and the products of inertia in the inertial tensor were obtained experimentally. A PD controller is also employed for rotational motion to compare with the proposed controller as described before. Feedback gains of the PD controller are determined by the pole assignment method. In this numerical simulation, the saturation values of the inputs $\delta_{a}, \delta_{e}$, $\delta_{r}$ and $T$ were $\pm 32[\mathrm{deg}], \pm 54[\mathrm{deg}], \pm 58[\mathrm{deg}]$ and $9.5[\mathrm{~N}]$, respectively. Table 1 shows the initial conditions of the simulation. The Dryden wind turbulence model is used for the disturbance, where the wind speed is set to be $2.5 \mathrm{~m} / \mathrm{s}$.

The results are shown in Figs. 7 - 9. The trajectories of the UAV are shown in Fig. 7. In comparison with the PD controller, the proposed flight control system can reach the desired state more smoothly and stably, as shown in Figs. 7, 8(a) and 9(a). Figure 9(c) shows that the attitude of the UAV cannot be controlled due to the effect of wind disturbance. To counter this effect, the transition flight is achieved using the proposed controller. Moreover, the control inputs of the UAV never exceed those limits, as shown in Figs. 8(c) and 8(d). These indicate that the proposed control system is suitable for the transitional flight.

\section{Experiment}

A flight experiment using a developed UAV was performed to confirm the effectiveness of the proposed flight control system.

\subsection{Experimental system}

Figure 6 shows the developed UAV, and its specification is shown in Table 1.

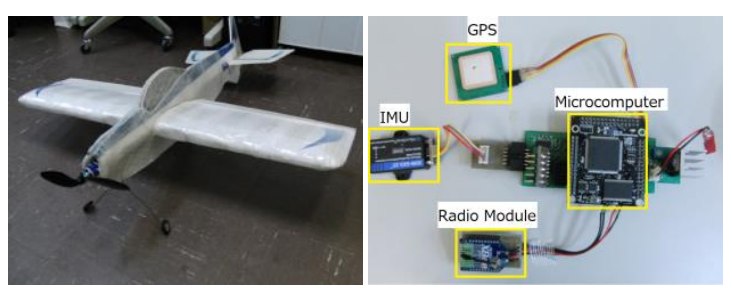

(a) Overview

(b) Avionics

Fig. 6. Developed UAV 


\section{R. HATORI et al.: Control Strategy of Transition Flight of a Fixed-wing UAV}

Table 1. Specification of the UAV.

\begin{tabular}{c|c|c}
\hline Mass $[\mathrm{kg}]$ & Wingspan $[\mathrm{m}]$ & Material \\
\hline 0.66 & 0.94 & EPP \\
\hline
\end{tabular}

\subsection{Results}

The target position was set as $\mathbf{x}_{c}=\left[\begin{array}{lll}30 & 0 & -30\end{array}\right]^{T}(\mathrm{~m})$. The wind speed was about $4(\mathrm{~m} / \mathrm{s})$. It can be seen from Figs. 10(a) and (b) that the positions of the UAV and target converged, and the attitude because stable when the UAV reached the target position. On the other hand, for the positions shown in Figs. 11(a) and (b), large steady-state errors occurred compared to the proposed system. The attitude because the PD controller did not consider the modeling error. Thus, the system that was used for comparison could not fulfill the transition.

\section{Conclusion}

In this paper, we proposed a robust flight control system that consists of translational and rotational controllers, which allow a fixed-wing UAV to transition from level flight to hovering. The numerical and experimental results confirm the effectiveness of the flight control system.

\section{References}

1) Hsiao, F., Ding, Y., Teng, Y, Chuang, C, Lin, C. and Huang, Y. The Design of a Small UAV System as a Testbed of Formation Flight, Infotech@Aerospace 2011, AIAA-2011-1422.

2) Cox, T. H., Nagy, C. J., Skoog, M. A. and Somers, I. A.: Civil UAV Capability Assessment -Draft Version-, NASA Technical Report, 2004.

3) Nonami, K.: Prospect and Recent Research \& Development for Civil Use Autonomous Unmanned Aircraft as UAV and MAV, Journal of System Design and Dynamics, 1 (2007), pp. 120-128.

4) Herwitz, S. R. et al,: Precision Agriculture as A Commercial Application for Solar-Powered Unmanned Aerial Vehicles, Proceedings of the AIAA 1st Technical Conference and Workshop on Unmanned Aerial Vehicles, 2002, AIAA- 2002-3404.

5) Cetinsoy, E., Dikyar, S., Hancer, C., Oner, K. T., Sirimoglu, E. Unel, M. and Aksit, M. F.: Design and Construction of a Novel Quad Tilt-wing UAV, Mechatronics, 22 (2012), pp. 723-745.

6) Lee, J., Min, B., and Kim, E.: Autopilot Design of Tilt-rotor UAV Using Particle Swarm Optimization Method, Proceedings of International Conference on Control, Automation and Systems, 2007, pp. 1629-1633.

7) Muraoka, K., Okada, N. and Kubo, D.: Quad Tilt Wing VTOL UAV: Aerodynamic Characteristics and Prototype Flight Test, AIAA Infotech@Aerospace Conference, 2009, AIAA-20091834

8) Matsumoto, T., Kita, K., Suzuki, R., Oosedo, A., Go, K., Hosihno, Y., Konno, A., and Uchiyama, M.: A Hovering Control Strategy for a Tail-Sitter VTOL UAV that Increases Stability Against Large Distance, Proceeding of IEEE International Conference on Robotics and Automation, 2010, pp.54-59.

9) Stone, R. H.: Control Architecture for a Tail-Sitter Unmanned Air Vehicle, Proceedings of the 5th Asian Control Conference, Vol.2 (2004), pp. 736-744.

10) Jung, Y., Shim, D. H. and Ananthkrishnan, N.: Controller Synthesis and Application to Hover-to-Cruise Transition Flight of a Tail Sitter UAV, AIAA, Atmospheric Flight Mechanics Conference, 2010, AIAA-2010-7508.

11) Myrand-Lapierre, V., Desbiens, A., Gagnon, E., Wong, F., and Poulin, E.: Transitions between Level Flight and Hovering for a Fixed-Wing Mini Aerial Vehicle, Proceedings of the American Control Conference, 2010, pp. 530-535.
12) Akai, Y., Shimada, Y., Uchiyama, K. and Abe, A.: Design of Nonlinear Attitude Control System for Spaceplane Using Disturbance-Accommodating Control, Proceedings of KSASJSASS Joint International Symposium on Aerospace Engineering, 2008, pp. 542-547.

13) Johnson, C.D.: A Family of "Universal Adaptive Controllers" for Linear and Nonlinear Plants, Proceedings of the Twentieth Southeastern Symposium on System Theory, 1988, pp. 530-534.

14) Giroux, R., Gurdeau, R., and Landry, R. J.: Extended Kalman Filter Implementation for Low-Cost INS/GPS Integration in a Fast Prototyping Environment, Proceedings of $16^{\text {th }}$ symposium on Navigation of the Canadian Navigation Society, 2005, pp. 1-11.

15) Iwasaki, K. and Uchiyama, K.: Robust Controller Design for Transition Flight for Fixed-Wing UAV, Proceedings of AsiaPacific International Symposium on Aerospace Technology, 2012 CD-ROM 7.5.2.

16) Kokume, M. and Uchiyama, K.: Control Architecture for Transition between Level Flight to Hovering of a Fixed-Wing UAV, Journal of JSASS, 60 (2012), pp. 173-180. 
Table 2. Conditions in numerical simulation.

\begin{tabular}{cccc}
\hline Initial attitude angles $\mathbf{q}_{0}$ & {$\left[\begin{array}{llll}0 & 0 & 0 & 1\end{array}\right]^{T}$} \\
Initial velocities $\mathbf{V}_{0}$ & {$\left[\begin{array}{lll}10 & 0 & 0\end{array}\right]^{T}$} \\
Initial angular rates $\mathbf{\omega}_{0}$ & {$\left[\begin{array}{lll}0 & 0 & 0\end{array}\right]^{T}$} \\
Initial positions $\mathbf{x}_{0}$ & {$\left[\begin{array}{lll}0 & 0 & 0\end{array}\right]^{T}$} \\
Position command $\mathbf{x}_{\mathrm{c}}$ & {$\left[\begin{array}{lll}60 & 30 & -60\end{array}\right]^{T}$} \\
\hline
\end{tabular}

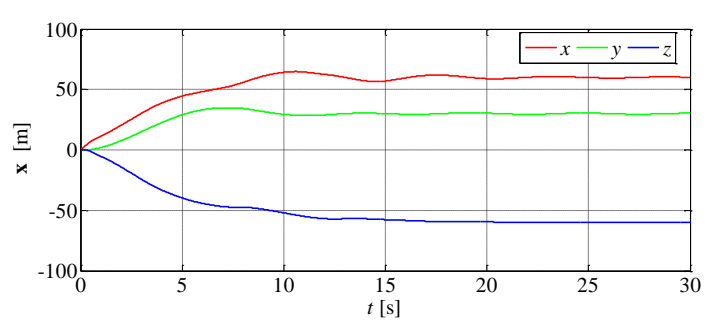

(a) Position

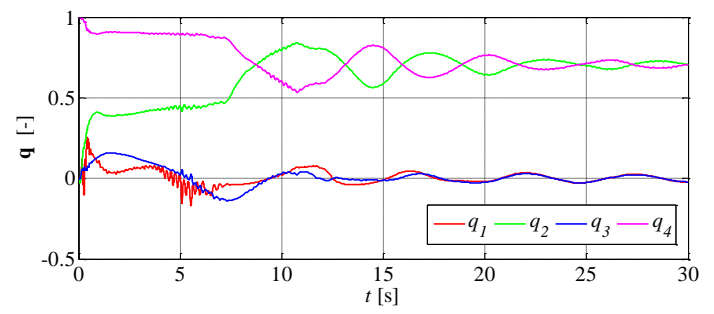

(b) Quaternion

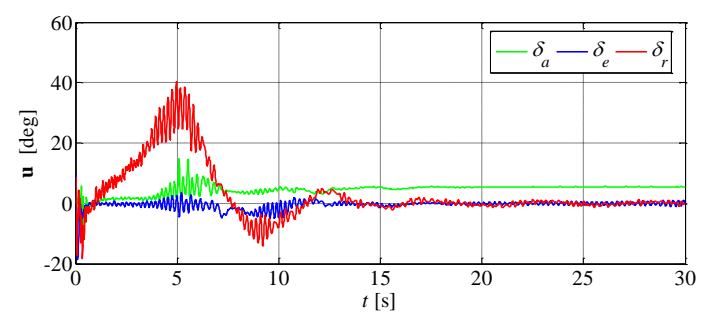

(c) Moving surfaces

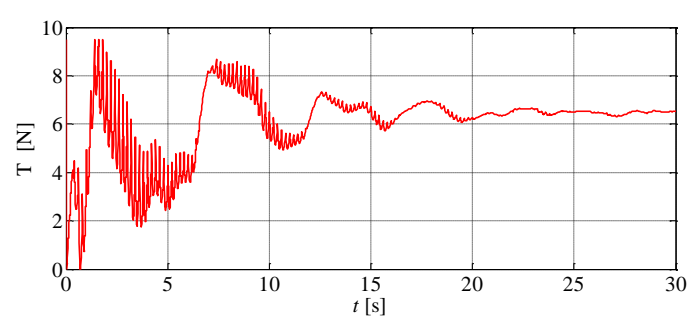

(d) Thrust

Fig. 8. Time responses of UAV using the proposed control system

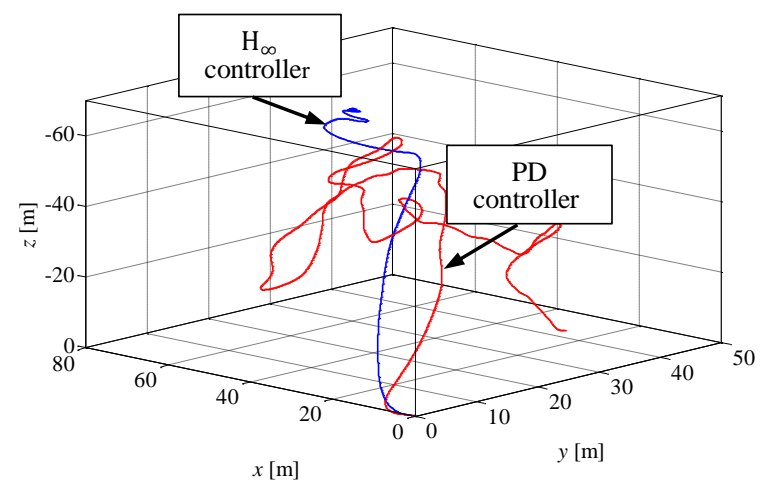

Fig. 7. Time histories of trajectory

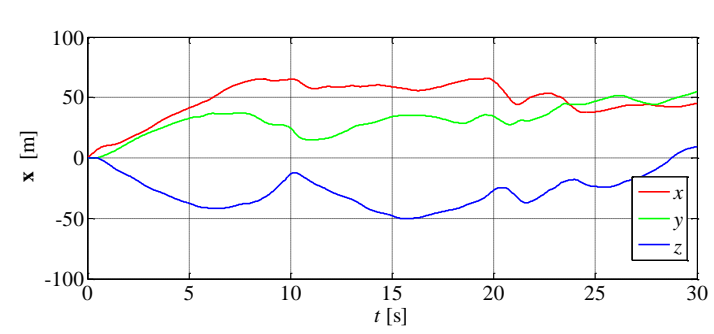

(a) Position

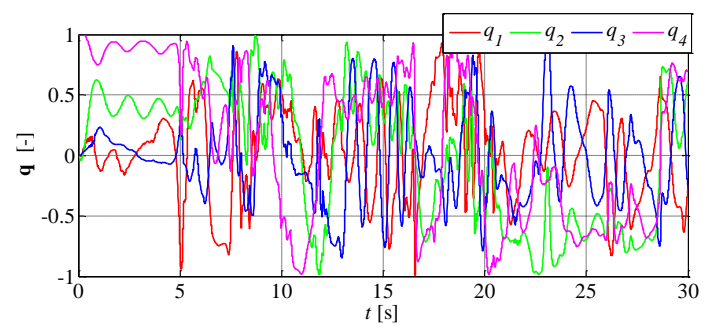

(b) Quaternion

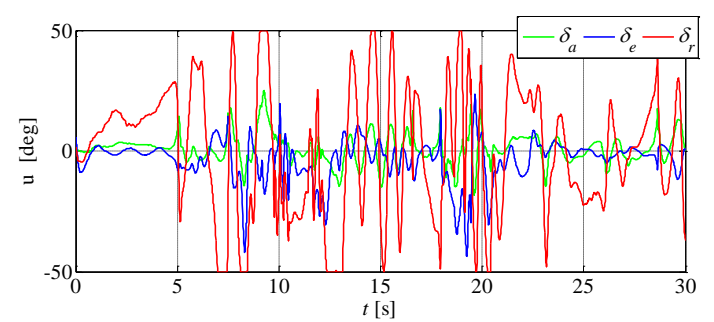

(c) Moving surfaces

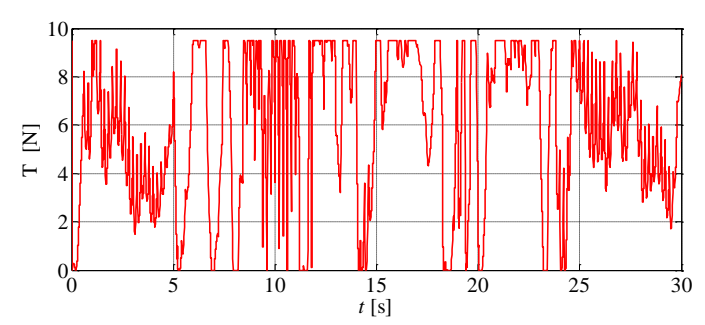

(d) Thrust

Fig. 9. Time responses of UAV using a PD controller 
R. HATORI et al.: Control Strategy of Transition Flight of a Fixed-wing UAV

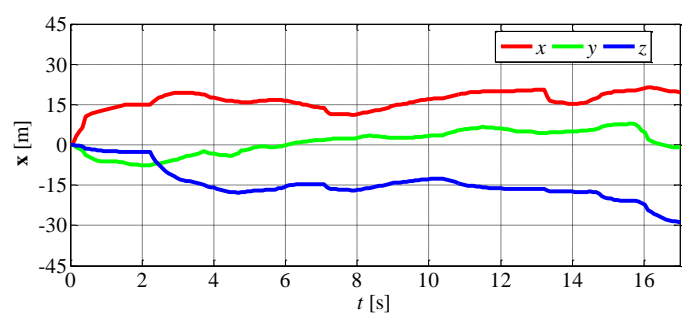

(a) Position

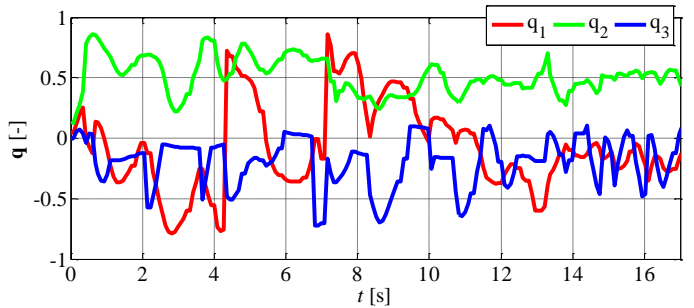

(b) Quaternion

Fig. 10. Time responses of developed UAV using the proposed control system

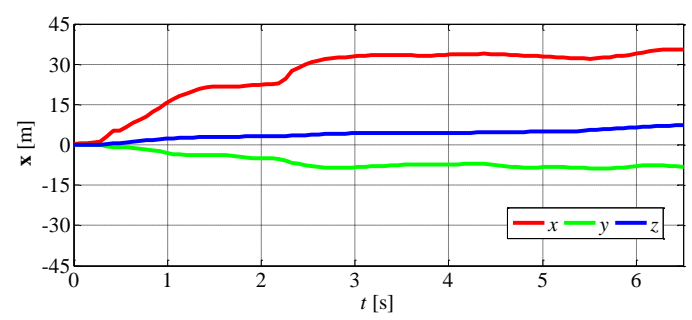

(a) Position

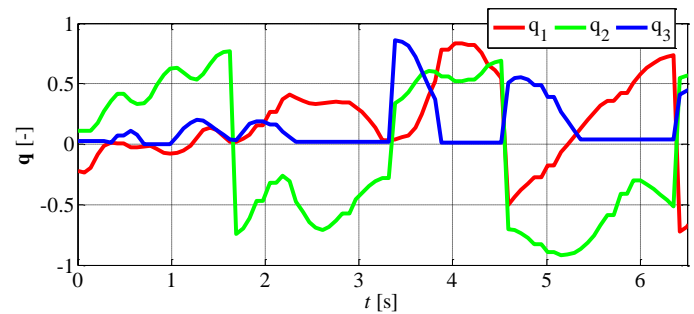

(b) Quaternion

Fig. 11. Time responses of developed UAV using a PD controller 